\title{
MÉTHODE DE PRISE DE SANG CHEZ LE PORC, A LA VEINE CAVE ANTÉRIEURE
}

P.AR

\author{
B.-L. DUMONT $\left({ }^{1}\right)$ \\ Station de Recherches sur l'Tlevage, C.N.R.Z., Jouy-en-Josas.
}

Au cours de l'étude de l'espace de diffusion de l'antipyrine chez le porc (I), nous avons été amenés à pratiquer sur un même animal, à de nombreuses reprises et à intervalle de temps assez court, des prises de sang veineux en quantité relativement importante (jusqu'à $50 \mathrm{~cm}^{3}$ à la fois).

Nous devions pratiquer en outre des mesures de l'espace de diffusion de l'antipyrine plusieurs fois dans la vie du sujet, pour en étudier les variations en fonction de l'âge et de l'état d'engraissement.

L'ensemble des conditions imposées aux prises de sang nécessaires à cette étude :

fréquence,

volume,

rapidité de la prise,

rend inutilisables les méthodes classiques de prises de sang chez le porc : prises à l'oreille, à la queue ou intracardiaque.

Par contre, la prise de sang à la veine cave antérieure, suggérée par CARLE et DrwhirsT (2), répond parfaitement à ces conditions. Toutefois, nous avons été amenés à modifier la méthode proposée par ces auteurs, dont l'emploi pour des porcs assez lourds s'est révélé délicat, sinon dangereux.

Il nous semble utile, étant donné les avantages de cette méthode et sa facilité d'exécution, d'indiquer avec précision les détails techniques et les règles pratiques de sa réalisation tels qu'ils résultent de l'utilisation systématique qui a été faite de cette méthode dans notre Station.

\section{Deseription de la méthode}

\section{Possibilités d'application}

La méthode est applicable aux porcs de différents poids. Elle a été surtout utilisée pour des porcs de 80 à Ioo $\mathrm{kg}$ vif.

(1) Cette méthode a été mise au point avec la collaboration technique de MM. Jean Wisır, Jean RFtTAGLIATI et Iaurice JAME. 


\section{Contention}

L'animal doit être immobilisé le mieux possible. Quel que soit le poids, la prise de sang s'effectue sur l'animal renversé et reposant sur le sol par les parties supérieures de l'épaule, des parois thoraciques et des parois dorsales (photo I).

Le mode opératoire est le suivant :

Un nœud coulant est serré autour de la mâchoire supérieure, derrière les crochets (photo 2). L'extrémité de la corde est reliée à un point fixe (anneau de mur ou traverse quelconque). En saisissant le bipède droit et en le tirant à lui d'un coup sec, un aide fait basculer le porc sur 1e dos (photo 3) et parfait ensuite la position du corps dans la position désirée : d'une part, par extension de la région cervicale, et d'autre part, par légère inclinaison du corps sur le côté. Eventuellement, pour compléter la contention de l'animal, on peut entraver les membres postérieurs et les relier à un point fixe, au sol. Cette condition n'est pratiquement pas nécessaire pour des porcs de moins de roo $\mathrm{kg}$.

L'aide enjambe le porc au niveau des dernières côtes et, écartant les deux membres antérieurs, présente à l'opérateur l'entrée de la poitrine et la région sternale de l'animal (photo 4).

\section{Localisation de la prise}

L'opérateur prend comme repère (photo 5) la ligne joignant la pointe du sternum à la base de l'oreille. L'aiguille est enfoncée sur cette ligne, environ à 3 ou $4 \mathrm{~cm}$ du sternum, point qui correspond en extérieur au défaut de l'épaule.

L'aiguille est enfoncée vers l'intérieur, vers l'arrière, très légèrement en oblique, vers le plan médian du corps. Elle atteint la veine cave antérieure dans l'arc costal compris entre la première et la deuxième côte (schéma I et II).

En raison de la position médiane de la veine cave antérieure, la prise est possible également du côté droit de l'animal.

\section{Matériel nécessaire}

Des aiguilles assez longues doivent être utilisées, leur longueur variant bien entendu avec la taille de l'animal (allant de $4 \mathrm{~cm}$ pour des porcs de $15 \mathrm{~kg}$ à $10 \mathrm{~cm}$ pour des porcs de $100 \mathrm{~kg}$ ). Le calibre de $10 \mathrm{~mm}$ convient parfaitement et évite des accidents possibles (dyspnée). 


\section{Quantité de sang}

Des quantités importantes de sang peuvent être prélevées rapidement à chaque prise. Une prise de $50 \mathrm{~cm}^{3}$ demande, pour un porc de $100 \mathrm{~kg}$, environ 20 secondes.

Des prises répétées ( 6 prises de $50 \mathrm{~cm}^{3}$ en deux heures) ont été effectuées à de nombreuses reprises, sans aucun dommage pour les animaux.

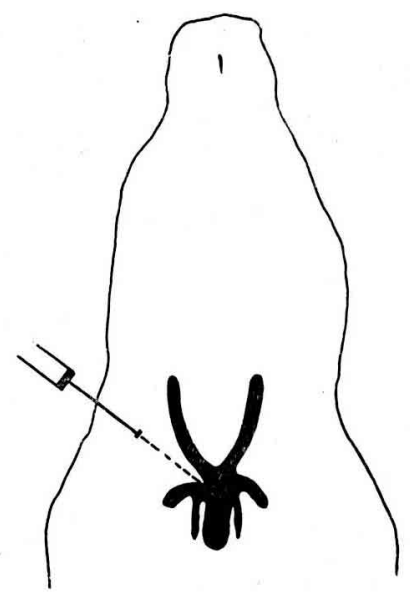

Schéma i. - Localisation de la prise. Vue da dessus (d'après Carle et Dewhirst (2)).

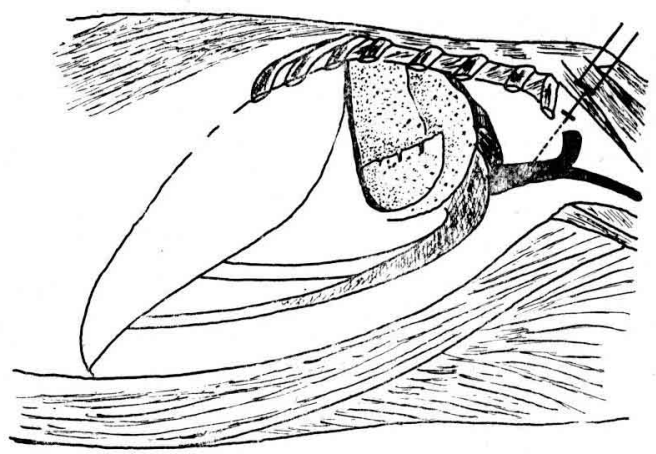

Schéma 2. - Localisation de la prise. Vue de côté (établie d'après Montané et Bourdelle, Anatomie du Porc).

\section{Réussite}

Sur de nombreux animaux expérimentaux destinés à l'abattage, nous avons pu pratiquer, après la mort, un examen de la carcasse et des organes au niveau de la région de l'entrée de la poitrine. Les prises de sang n'entraînent aucune altération dans cette région. 


\section{Discussion}

Cette méthode de prise de sang est extrêmement simple et facile à exécuter par deux personnes (l'opérateur et son aide). Sa pratique s'acquiert très rapidement. Contrairement à ce qui a été avancé par CARLE et DEWHIRST (2), il est très facile de pratiquer les prises de sang sur de gros porcs en position dorsale, leur contention étant relativement aisée, lorsqu'on adopte notre procédure. Bien au contraire, nous déconseillons formellement les prises sur animal debout et retenu simplement par une attache nasale (photo 6). D'une part, le travail de l'opérateur est malaisé et, d'autre part, la simple contention nasale permet à l'animal de remuer, soit latéralement, soit d'arrière en avant; car un pourcentage élevé des porcs (environ I/3) ne " tirent pas au renard " systématiquement, lorsqu'ils sont attachés par le nez. Des déplacements imprévus du porc risquent de compromettre la prise de sang et, d'autre part, de provoquer de graves accidents chez l'animal (hématomes) qui, sans entraîner nécessairement la mort, interdisent des prises de sang ultérieures.

Cette méthode permet en outre des prises de sang stérile en quantités importantes et des injections rapides de solutions.

Eille s'avère donc d'un grand intérêt dans diverses applications, tant dans la pratique vétérinaire que dans le domaine de la Recherche.

\section{RÉFÉRENCES BIBLIOGRAPHIQUES}

(I) Dumont (B.-L.). - Annales de Zootechnic, I955, IV, p. 305-313.

(2) CARLE (B. N.) et DEWhirst. - Jour. A.V.M.A., p. 495-96, I942. 
(IV, I955)

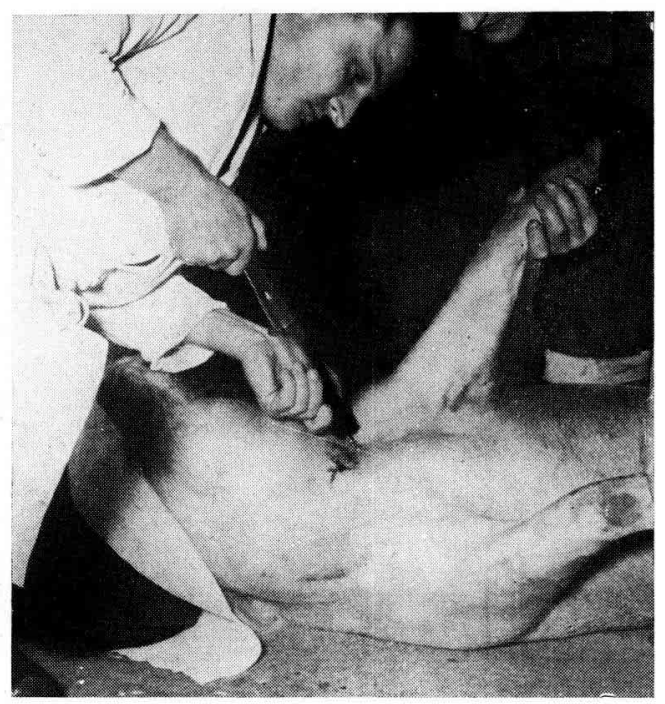

Photo I

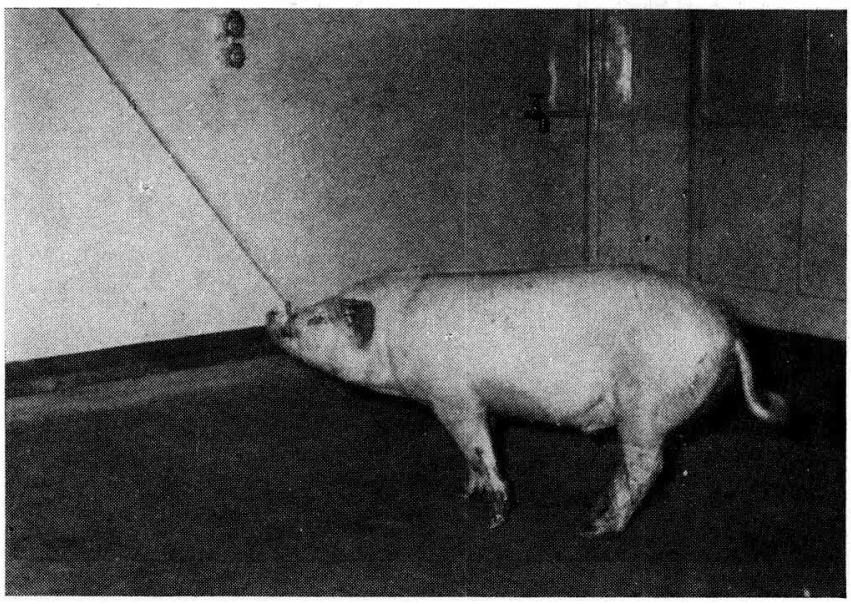

Photo 2 


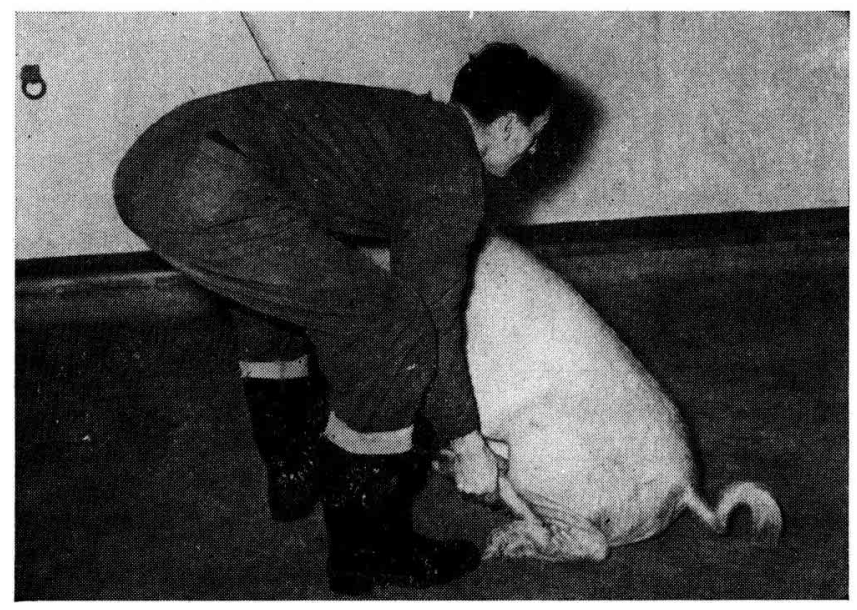

Photo 3

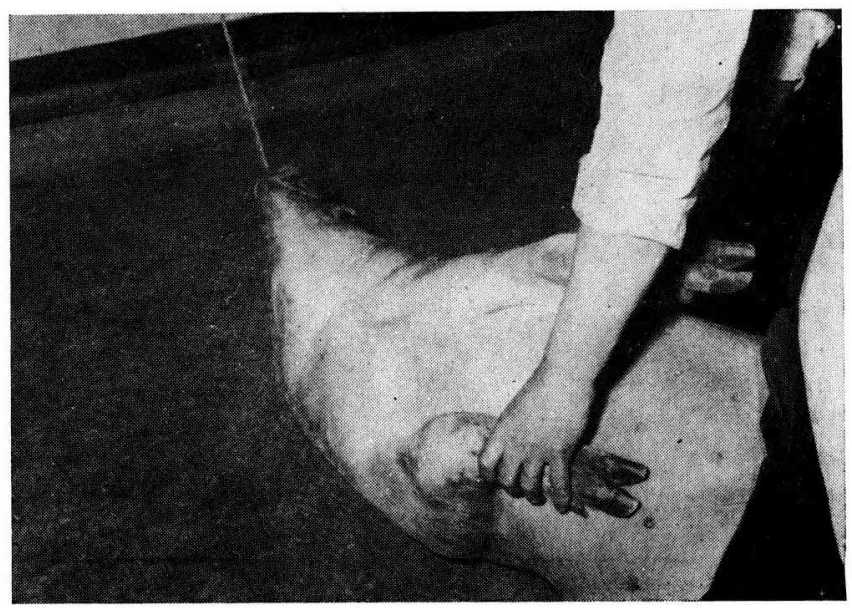

Photo 4 


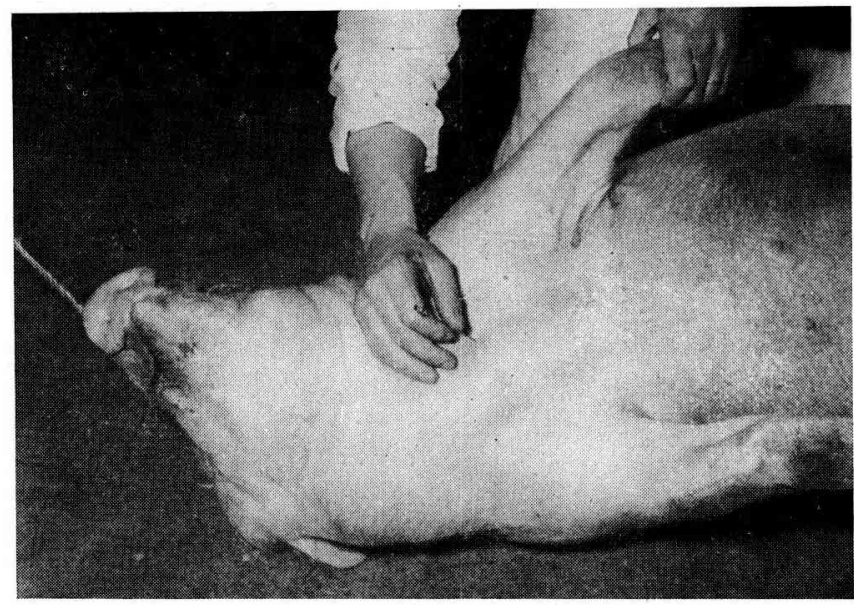

Photo 5

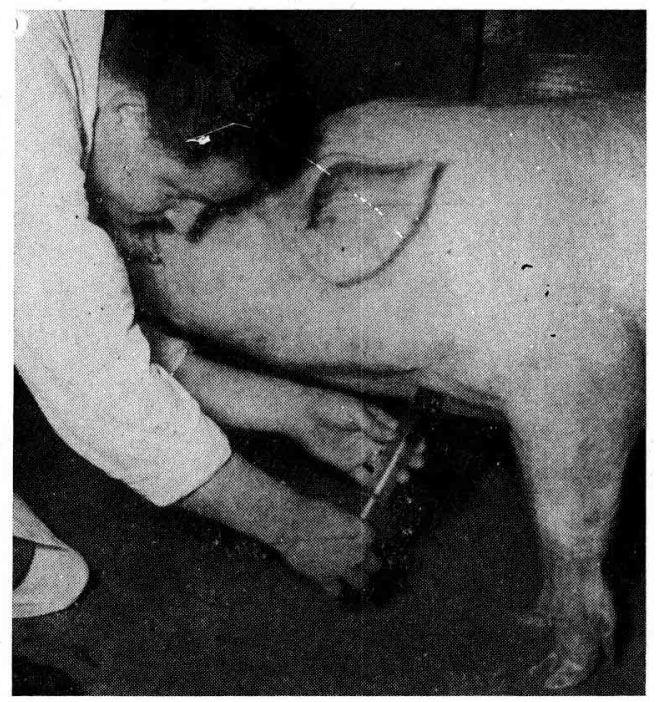

Photo 6 